\title{
Hyper Infection of Strongyloides Stercoralis Associated With Secondary Bacterial Pneumonia in a Malnourished Patient
}

\author{
Raghu Prakash Reddy Nayakanti ${ }^{1}$, Rama Kesava Reddy Motati $^{2}$, Nettikantaiah Muneppagari ${ }^{3}$, \\ Mohanraj Rathinavelu Mudhaliar ${ }^{4}$
}

${ }^{1}$ Medical Microbiologist, Rural Development Trust (RDT) Hospital, Bathalapalli, Ananthapuramu, Andhra Pradesh, INDIA ${ }^{2}$ Consultant Physician, Department of General Medicine, Rural Development Trust (RDT) Hospital, Bathalapalli, Ananthapuramu, Andhra Pradesh, INDIA

${ }^{3}$ Microbiologist, Rural Development Trust (RDT) Hospital, Bathalapalli, Ananthapuramu, Andhra Pradesh, INDIA

${ }^{4}$ Drug Information Pharmacist, Poison and Drug Information Center (PDIC), Rural Development Trust (RDT) Hospital, Bathalapalli, Ananthapuramu, Andhra Pradesh, INDIA

\begin{abstract}
Strongyloides stercoralis, an intestinal nematode is more prevalent in the tropics or subtropics. Hyper infection syndrome in immuno competent patients is due to asymptomatic infections, increased parasite number, dissemination and it leads to death if unnoticed. Use of corticosteroids, HIV, HTLV-1, malignancies and malnutrition are predisposing factors for hyper infection. Secondary bacterial infections are known to cause increased mortality in these patients in antibiotic resistant era. Here we report a case of strongyloides hyper infection syndrome associated with secondary bacterial pneumonia in a malnourished female patient.
\end{abstract}

Key words: Hyper infection syndrome, Immune compromised, Malnourished, Pneumonia, Strongyloides stercoralis.

\section{INTRODUCTION}

Strongyloidosis, an intestinal nematode infection is prevalent in the tropical and subtropical regions especially in low socio-economic countries where the faecal contamination of soil or water is common. The most common source of infection is by contacting soil that is contaminated with Strongyloides larvae. Therefore, activities that increase contact with the soil such as walking with bare feet, contact with human waste or sewage, occupations that increase contact with contaminated soil such as farming and coal mining, increase the risk of becoming infected which are all being commonly practiced in India. ${ }^{1}$

Strongyloides infection in immunocompetent individual is usually asymptomatic. In the immunocompetent host, the immune system regulates the population density of adult worms in the intestine. ${ }^{2}$
Immunocompromised status is a predisposing factor for hyper infection. In the era of increased use of corticosteroids and anticancer drugs there is an absolute chance for developing hyper infection syndrome in a previously infected and asymptomatic patient.

Autoinfection, hyper infection and disseminated infections are the three related entities shown by the parasite during its course of infection. There is no clear demarcation between autoinfection and hyper infection where the hyper infection is an exaggerated mechanism of autoinfection along with clinical signs and symptoms related to GIT and pulmonary system. Increased numbers of larvae in stool and/or sputum is the hallmark of hyper infection. Whereas in disseminated infection there is a deviation of the course of larva from its routine
DOI: 10.5530/ijopp.10.2.32

Address for correspondence: Dr. Raghu Prakash Reddy Nayakanti. M.D, Medical Microbiologist, Rural Development Trust (RDT) HOSPITAL, Bathalapalli, Ananthapuramu, Andhra Pradesh, INDIA. Phone no: +91 9490419834 E-mail: raghusvims@gmail.com

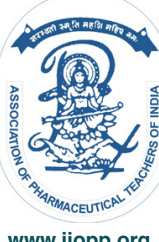

www.ijopp.org 
cycle and it enters into various organs systems like brain, kidneys etc causing devastating consequences. ${ }^{3}$ Auto-infective larvae can carry gut bacteria to the other parts of the body. Many of the people with hyper infection present with bacterial disease due to enteric bacteria. Case reports of bacteremias and meningitis with the enteric bacteria like Escherichia coli and Klebsiella have been reported. ${ }^{4}$ The high mortality rate associated with hyper infection syndrome and disseminated disease is frequently due to secondary bacterial infections. ${ }^{5}$

Herewereportacase of hyperinfectionsyndromeleading to secondary bacterial pneumoniacaused by mixed infection of Klebsiella and Pseudomonas.

\section{CASE REPORT}

Female patient aged 45 years presented to the hospital with severe breathlessness and right sided chest pain associated with 2 episodes of hemoptysis in the morning. She had a history of cough since 2 months, associated with intermittent productive sputum along with shortness of breath and right sided chest pain which increased in the intensity for the last 15 days and became intolerable on the day of presentation to the hospital. She was admitted and started with empirical antibiotic therapy.

Patient is not a known case of DM and HTN. She had past h/o pneumonia 8 months back which was relieved after the treatment. No past h/o pul TB / asthma/ CAD/CVA

A panel of Investigations was done to identify the underlying cause.

Sputum was sent for Gram's staining and acid fast staining. No Acid fast bacilli were observed in Aura mine staining and the Gram's stain showed plenty of neutrophils along with many gram negative bacilli. And round coiled hollow structures appearing like larvae were also observed surrounded by neutrophils.

Wet mount of the sputum was made and plenty of motile larval forms (3-4 parasites/ LPF) corresponding with the morphology of filariform larvae were observed.

Culture of the sputum was done on blood agar and McConkeys agar.

$\mathrm{X}$ ray and other routine blood investigations were done.

$\mathrm{X}$ ray chest showed diffuse infiltrations in the right lung with consolidation of right lower lobe. Blood investigations showed significant neutrophilia with

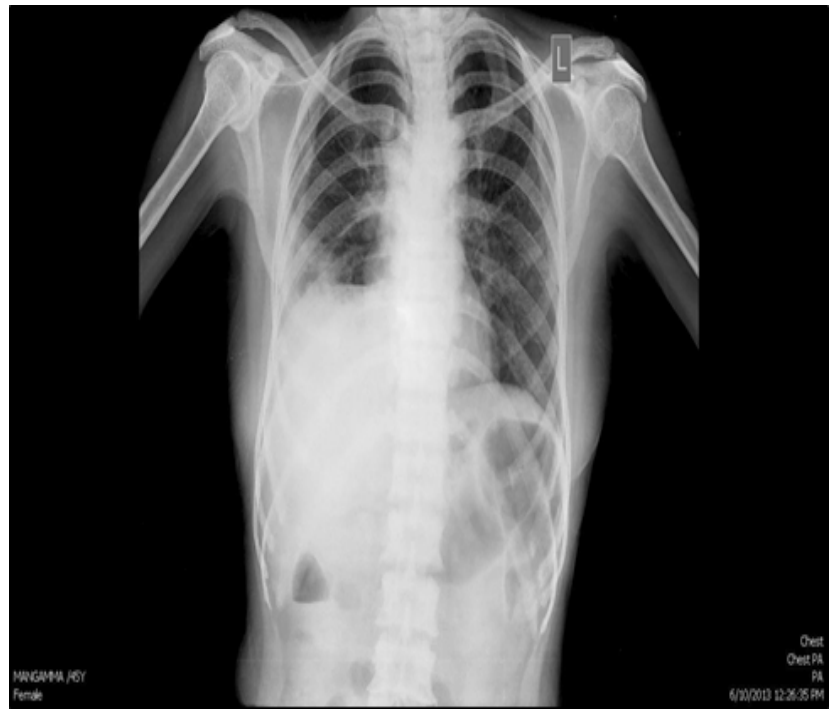

Figure 1: Diffuse infiltrations in the right lung with consolidation of right lower lobe (Chest X-ray PA view).

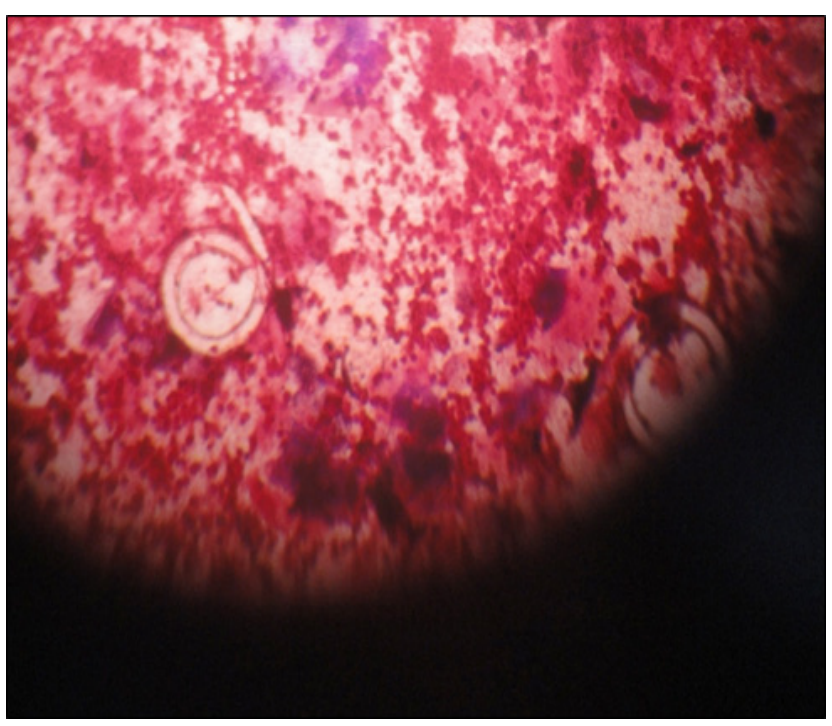

Figure 2: gram staining of sputum showing larvae of strongyloides stercoralis.

$>85 \%$ of WBC being neutrophils. Ig E levels were slightly elevated.

Clinician was informed about the larvae and the patient was started with additional ivermectin treatment. Next day stool was asked for wet mount examination and observed many sluggishly motile and some non-motile larvae of strongyloides stercoralis. Blood agar plate also showed serpiginous markings along with bacterial colonies because of the surface movements of the larvae on the agar plate.

Two types of colonies were isolated from the sputum. The predominant organism was Pseudomonas and the other organism being Klebsiella. Antibiotic 
sensitivities were determined for both the organisms.

Empirical antibiotic therapy was changed to ciprofloxacin after receiving the sensitivity report as both the organisms were sensitive to it. Patient recovered uneventfully in course of time and the pneumonia was resolved.

\section{DISCUSSION}

In the present case, though the exact immunosuppressive condition could not be made out with the available clinical and diagnostic measures, we cannot rule out the hidden foci of such conditions which may be expressed out in course of time. In this case the preference is given to the malnutrition status of the patient which might have played a role in the causation of hyper infection. And the presence of Pseudomonas super infection indicates an underlying compromised status as this bacterium is a known opportunistic pathogen.

There is quiet a high chance of misdiagnosing the hyper infection with the bacterial pneumonias, SLE and other inflammatory conditions. As there are only very few case reports of this condition came as literature in Indian context, clinicians rarely suspect this condition and they may not even include it in their routine differential diagnosis panel. At least in endemic areas of S. stercoralis, strongyloidiasis should be included as a possible cause of pulmonary disease in differential diagnoses, especially in patients with immunodeficiencies and abnormal chest imaging findings, like alveolar and interstitial shadow patterns or lobar pneumonia. ${ }^{6}$

High suspicion and thorough clinical and microbiological examination is necessary to identify this condition and to treat the patient with appropriate therapy otherwise the patient condition will be deteriorated which ultimately leads to bad outcome.

\section{ACKNOWLEDGEMENT}

The authors would like to thank Dr. Gerardo Alvarez-Uria, Head - Care and Supportive Center, Rural Development Trust (RDT) Hospital, Bathalapalli, Ananthapuramu, Andhra Pradesh for his constant support and guidance throughout the study.

\section{CONFLICT OF INTEREST}

The author declares no conflict of interest.

\section{FUNDS AND GRANTS}

Nil

\section{ABBREVIATIONS}

HIV: Human immunodeficiency virus; HTLV-1: Human T cell lymphotropic virus type 1; GIT: Gastro Intestinal Tract; DM: Diabetes Mellitus; HTN: Hypertension; TB: Tuberculosis; CAD: Coronary Artery Disease; CVA: Cerebro Vascular Accident; Ig: Immunoglobulin; LPF: Low Power Field; SLE:Systemic Lupus Erythematosus; WBC: White Blood cell Count; PA: Posteroanterior.

\section{REFERENCES}

1. CDC. Parasites-Strongyloides: epidemiology and risk factors. Atlanta, GA: US Department of Health and Human Resources, CDC; 2012.

2. Genta, RM. Strongyloidiasis. In: Guerrant, RL. Editor. Tropical Infectious Diseases. Philadelphia: Elsevier; 2005. p. 1274-82.

3. Ramanathan R, Nutman TB. Strongyloides stercoralis infection in the immunocompromised host. Current Infectious Disease Reports. 2008;10(2):105-10. https://doi.org/10.1007/s11908-008-0019-6; PMid:18462583 PMCid:PMC3401551.

4. Keiser PB, Nutman TB. Strongyloides stercoralis in the immunocompromised population. Clinical Microbiology Reviews. 2004;17(1):208-17. https://doi. org/10.1128/CMR.17.1.208-217.2004; PMCid:PMC321465.

5. Link K, Orenstein R. Bacterial complications of strongyloidiasis: Streptococcus bovis meningitis. Southern Medical Journal. 1999;92(7):728-31. https://doi. org/10.1097/00007611-199907000-00016; PMid:10414486.

6. Jayaprakash B, Sandhya S, Kumari AK. Pulmonary strongyloidiasis. J Assoc Phys India. 2009;57:535-36 PMid:20329418. 